Background and Aim Injury due to foreign body (FB) aspiration and/or ingestion is a common and serious pediatric emergency. FB injury (FBI) most commonly occurs in children less than six years, and the incidence of FBI has increased in recent years. The aim of the present study was to evaluate and compare the characteristics of FBI due to ingestion and aspiration.

Methods Data from who were hospitalized for FB ingestion and/ or aspiration and underwent rigid bronchoscopy and esophagoscopy from 2008-2011 were retrospectively evaluated. FB in the upper aerodigestive tract (ADT) was evaluated with respect to the characteristics of patients, clinical presentation, management strategy, the outcome, and features of FB.

Results A total of 192 patients admitted for FB ingestion or aspiration in the pediatric surgery department were evaluated. The mean age was $40.97 \pm 35.73$ months. The majority of patients were fewer than four years of age. FB were mainly located in the upper esophagus for ingested FB $60.8 \%$, while for aspiration $43 \%$ of FB were in the main right bronchus. A total of four patients died. The hospitalization period of patients admitted for FB aspiration was longer than that of patients with FB ingestion. Surgery was performed in four patients. The most commonly ingested FB were coins, while seeds were the most commonly aspirated.

Conclusion Prevention is the key to dealing with FBI. Since the frequency of foreign bodies is higher in under-developed countries, education of parents regarding the dangers and prevention of aspiration and ingestion is important.

\section{TIME OF DAY AS A DETERMINANT OF RADIOGRAPHIC IMAGING IN THE EVALUATION OF PEDIATRIC INTUSSUSCEPTION}

doi:10.1136/archdischild-2012-302724.1491

'YJ Chang, ${ }^{2} \mathrm{CT}$ Wu, ${ }^{2} \mathrm{JL}$ Huang, ${ }^{2} \mathrm{HC}$ Chao. ${ }^{1}$ Pediatrics; ${ }^{2}$ Chang Gung Memorial Hospital, Chang Gung University College of Medicine, Taoyuan, Taiwan R.O.C.

Background Although ultrasound is often the preferred pediatric imaging study, many institutions lack ultrasound access at night for evaluation of intussusceptions in children. The purpose of this study was to characterize patterns of daytime and nighttime use of radiographic imaging for evaluation of intussusceptions.

Methods A retrospective chart review of patients evaluated for intussusceptions from January 2010 to December 2010 was performed to evaluate daytime and nighttime use of radiographic imaging for pediatric patients. Patients were further stratified by time of performance of imaging study into "day" and "night" categories. Differences in clinical characteristics, imaging study, cost, and final diagnosis between daytime and night were analyzed.

Results 86 pediatric consults with suspected intussusceptions were performed. Forty (46.5\%) consults were performed during the day. During the day, 38 (95\%) patients underwent US and 33 (82.5\%) had abdominal films. At night, 3 (6.5\%) CTs and 39 (84.7\%) abdominal films were performed. The positive rate of enemas during the daytime was significant statistically higher than at night $(97.6 \%$ vs $59.2 \%$, p $<0.001)$. Average daytime cost of initial radiology evaluation was 1159 dollars compared with 3328 dollars at night $(p<0.001)$. In terms of radiation dose, the daytime dose was significantly lower than the those at night $(0.63 \pm 0.48 \mathrm{mSv}$ vs $2.06 \pm 1.48$ $\mathrm{mSv}, \mathrm{p}<0.001$ )

Conclusions Radiographic imaging at night results in higher average radiation exposure and cost. Twentyfour-hour ultrasound availability would decrease radiation exposure and cost of evaluation of children presenting with intussusceptions.

\section{SOCIODEMOGRAPHIC FACTORS INFLUENCE THE RISK FOR FEMUR SHAFT FRACTURES IN CHILDREN: A SWEDISH CASE-CONTROL STUDY FROM 1997-2005}

doi:10.1136/archdischild-2012-302724.1492
IJ von Heideken, ${ }^{2} \mathrm{~T}$ Svensson, ${ }^{3,4} \mathrm{M}$ Iversen, ${ }^{2} \mathrm{P}$ Blomqvist, ${ }^{2} \mathrm{Y}$ Haglund-Åkerlind, ${ }^{1} \mathrm{PM}$ Janarv. 'Department of Women's and Children's Health; ${ }^{2}$ Department of Medicine, Solna, Clinical Epidemiology Unit, Karolinska Institutet, Stockholm, Sweden; ${ }^{3}$ Department of Physical Therapy, Northeastern University; ${ }^{4}$ Division of Rheumatology, Immunology and Allergy, Brigham and Women's Hospital, Boston, MA, USA

Objectives To investigate gender and age differences in sociodemographic risk factors and their relationship with femur shaft fractures and injury mechanisms in children.

Methods Population based case-control study. Swedish children $(\mathrm{N}=1,874)$, aged $0-14$ years, with a femur shaft fracture diagnostic code between 1987-2005 were compared to matched controls $(\mathrm{N}=18,740)$. Data were based on record linkage between six Swedish registers. Adjusted Odds Ratios were calculated.

Results Parental age $<25$ years old increased the risk (25\%) for fracture, compared to parents with an average age of 25-37 years. When stratifying for gender and age group, the risk (40\%) was only seen in older boys, 7-14 years of age. If parents' total income was among the $25^{\text {th }}$ percentile, the risk $(20 \%)$ increased, compared to parents with an income in the $50^{\text {th }}$ percentile. The risk $(50 \%)$ was only seen in older girls living in low-income households. Children with at least one university-educated parent reduced their fracture risk (15\%), compared to children whose parents had 10-12 years of education, but this decrease could not be linked to gender and age group.

Family composition, number of siblings, birth order or receiving social welfare did not influence the fracture risk.

Regarding the cause of injury none of the sociodemographic variables influenced the risk equal for boys and girls.

Conclusions Sociodemographic differences related to femur shaft fracture rate and cause of injury differ between boys and girls in different age groups. This have implications for parental counselling.

\section{CD 64 AS A DIAGNOSTIC MARKER OF A SERIOUS BACTERIAL INFECTION IN CHILDREN WITH FEVER PRESENTING TO THE EMERGENCY DEPARTMENT}

doi:10.1136/archdischild-2012-302724.1493

${ }^{1} \mathrm{M}$ van Veen, 'R Nijman, ${ }^{2} \mathrm{M}$ Zijlstra, ${ }^{1} \mathrm{H}$ Moll, ${ }^{2} \mathrm{~F}$ Smit, ${ }^{1} \mathrm{R}$ Oostenbrink. 'Erasmus MC - Sophia Children's Hospital; '2Paediatrics, Maasstad Hospital, Rotterdam, The Netherlands

Background and Aims Expression of neutrophile CD64 is elevated in presence of a bacterial infection. Studies on its diagnostic value show good results in adults, and diverse results in neonates and children admitted to the ICU with sepsis. We aimed to determine the diagnostic value of CD64 in children presenting with fever at the emergency department (ED), to detect a serious bacterial infection (SBI).

Methods We performed a prospective observational study including children aged 1 month-16 years with fever, who presented to the ED of a large teaching hospital in the Netherlands. Patients were excluded in case of relevant comorbidity. CD64 was determined using flowcytometry. SBI was assessed based on a combination of a positive culture, radiology or consensus diagnosis and included disease course follow up by an inpatient visit or telephonic follow up.

Results During 6 months in 2011 we included 208 children of whom CD64 was determined in 137 patients (66\%). Preliminary results are based on 137 febrile children, of which 28 (20.4\%) with a SBI. The area under the curve of the receiver operator curve to assess SBI was 0.65 (95\% CI 0.53-0.77).

Conclusion In a sample of febrile children at the $\mathrm{ED}$, we assessed moderate diagnostic value of CD64 as marker of a SBI. Our further analysis on complete sample (estimated $n=280$ ) will include diagnostic performance of CD64 for specific bacterial and viral diagnoses. 